\title{
NeurOMP: Paralelização automática de código utilizando Aprendizagem por Reforço
}

\author{
João Saffran ${ }^{1}$, Rodrigo Caetano O. Rocha ${ }^{2}$, Luís Fabrício W. Góes ${ }^{3}$ \\ ${ }^{1}$ Departamento de Ciência da Computação \\ Universidade Federal de Minas Gerais \\ ${ }^{2}$ Institute for Computing Systems Architecture (ICSA) \\ University of Edinburgh \\ ${ }^{3}$ Instituto de Ciência Exatas e Informática \\ Pontifícia Universidade Católica de Minas Gerais
}

joaosaffran@ufmg.br,r.rocha@ed.ac.uk, lfwgoes@pucminas.br

\begin{abstract}
Building high performance applications is a daunting task. The programmer must be aware of several application development components, including parallelism techniques. Therefore modern compilers try to modify applications code to explore parallelism automatic, using static code analysis. One way to improve this strategy is using Reinforcement Learning (RL). This paper proposes a new compiler transformation, called NeurOMP, which uses RL to parallelize for loops in $C$ code automatically using the OpenMP library. Experimental results showed that NeurOMP has an average speedup of 1.6 on CAP Bench, similar to a human specialist.
\end{abstract}

Resumo. A criação de aplicações que obtenham o máximo de desempenho computacional nas arquiteturas modernas é uma tarefa complexa. Além de utilizar conhecimentos de paralelismo, o programador precisar ter um amplo conhecimento de vários outros aspectos da aplicação. Por este motivo, os compiladores modernos tentam paralelizar algoritmos de maneira automática, utilizando a análise estática do código. Uma maneira de melhorar o processo de tomada de decisão do compilador é utilizando Aprendizagem por Reforço (RL). Este trabalho propõe e avalia uma otimização, chamada NeurOMP, que utiliza $R L$ para paralelizar for loops em códigos $C$ utilizando a biblioteca OpenMP. Os resultados experimentais mostram que o NeurOMP obtém um speedup médio no CAP Bench de 1.6, similar a um especialista humano.

\section{Introdução}

O paralelismo se tornou um componente essencial em sistemas de computação, aplicações e algoritmos modernos [Ahmed et al. 2017]. A criação de aplicações escaláveis que obtenham o máximo de desempenho exige que o desenvolvedor conheça amplamente da arquitetura, do hardware e do compilador que ele utiliza, além de envolver o conhecimento da aplicação, algoritmos e estruturas de dados envolvidas na solução do problema. Devido a esta complexidade no desenvolvimento de uma aplicação paralela, os compiladores vem se preocupando cada vez mais em otimizar o código fonte, visando evitar que o programador tenha que paralelizar o código explicitamente [Schardl et al. 2017] [Tong et al. 2017]. 
As otimizações do compilador envolvem várias transformações e mudanças no código de entrada, com o objetivo de remover, adicionar e alterar operações [Triantafyllis et al. 2003]. Um processo que os compiladores tentam automatizar é o processo de paralelização [Moreira et al. 2016]. Isto vem sendo amplamente pesquisado, onde várias técnicas e ferramentas já foram desenvolvidas para tornar esse processo menos custoso para os programadores. Alguns exemplos são: i) criação de bibliotecas que ajudem o compilador no processo de paralelização, como por exemplo o OpenMP [Dagum and Menon 1998], juntamente com uma API que permite ao programador paralelizar o código utilizando diretivas de compilação; ii) transformações de código usadas para paralelizar o código através da análise estática do código [Bessey et al. 2010].

Estes avanços facilitam o processo de paralelização de código, mas ainda assim este problema não foi resolvido [Chapman et al. 2008]. Os conceitos envolvidos na paralelização de código são complexos para uma parte dos programadores. Um outro aspecto impactante é que essas bibliotecas necessitam que os programas sejam reescritos para utilizá-las no processo de paralelização. Este mesmo problema afeta o compilador na hora de realizar a análise estática. Para que o compilador obtenha a paralelização que causa a maior redução do tempo de execução, várias outras transformações intermediárias devem ser realizadas [Khatami 2017].

O compilador tem um outro empecilho quando realiza as transformações. Uma vez que um programa tem um comprometimento com a corretude e desempenho do código gerado, este fator força o compilador a se comportar de maneira conservadora. Um exemplo disso é quando o compilador faz a vetorização (processo de paralelização de instruções realizado pelo compilador) [Gubner and Boncz 2017]. Se o custo de um conjunto de instruções for baixo, o compilador pode não vetorizar essas instruções, acreditando que essa vetorização não garante nenhum ganho. A solução mais adotada para esse problema é permitir que o programador determine manualmente qual o custo mínimo para que a transformação ocorra [Coelli 1996].

Uma maneira de melhorar as otimizações do compilador seria utilizar algoritmos de inteligência artificial (IA) [Stephenson et al. 2003]. Apesar de não serem tão assertivas quanto a análise estática, estas técnicas ajudam no processo de tomada de decisão do compilador, tornando-o capaz de criar heurísticas mais eficientes que o ajuda a criar códigos com melhor desempenho computacional.

Um campo de IA promissor para ser aplicado dentro do processo de compilação é a Aprendizagem por Reforço (RL) [Kulkarni and Cavazos 2012]. Este campo estuda a criação de agentes inteligentes capazes de tomar decisões em um ambiente, que pode ser estocásticos ou determinísticos, com o propósito de maximar o acúmulo de recompensa a longo prazo.

Uma das vantagens de RL é utilizar um modelo minimalista do mundo. Com um Processo de decisão de Markov [Davis 2018], um arcabouço matemático utilizado para modelar problemas de RL, é possível resolver problemas apenas observando as recompensas recebidas pelas ações tomadas pelo agente.

Um algoritmo capaz de fazer o acúmulo de recompensas em longo prazo é o QLe$\operatorname{arning}$ [Mnih et al. 2013]. Este algoritmo descobre uma função que determina uma distribuição de probabilidade para as ações do agente, sofrendo alterações dependendo do 
estado do agente e do mundo. Desta forma obtêm-se qual a melhor ação para cada estado do mundo.

O objetivo deste trabalho é a criação de uma otimização que utiliza Aprendizagem por Reforço para fazer a paralelização automática de código, loops do tipo for, utilizando OpenMP. Além de implementar a transformação, compara-se o resultado desta otimização com as já existentes e com um especialista em paralelismo. Para mensurar isto, utiliza-se o CAP Bench, um benchmark [Souza et al. 2017] já paralelizado com OpenMP e, além disso, são comparados os processos de análise estática para geração automática de código dos compiladores ICC e GCC.

O restante deste trabalho está dividido em 7 Seções. A seção 2 discute os trabalhos relacionados. Seções 3 e 4 explicam conceitos de Computação Paralela e Aprendizagem por Reforço, respectivamente. O processo utilizado pela otimização é apresentado na Seção 5. A Seção 6 apresenta os resultados experimentais. Por fim, na Seção 7 são apresentadas as conclusões deste trabalho.

\section{Trabalhos Relacionados}

Nesta seção, são discutidos os trabalhos relacionados. Os conceitos abordados nessa seção são: Aplicações de Aprendizagem de Máquina dentro do processo de compilação e técnicas de paralelização automática de código.

Devido à constante evolução das arquiteturas dos computadores modernos, definir os parâmetros usados nas otimizações internas do compilador se torna uma tarefa complexa para ser realizada por seres humanos. Por isso, o trabalho [Fursin et al. 2008] propõe o uso de Aprendizagem de Máquina (AM) para que o compilador aprenda automaticamente quais os melhores parâmetros para as heurísticas utilizadas no compilador para cada arquitetura.

No trabalho [Moreira et al. 2016], os autores utilizam análise estática para realizar a paralelização do código de maneira automática utilizando diretivas do OpenACC. Este trabalho não utiliza AM para tentar paralelizar o código, ao invés disso, são apresentadas várias regras criadas manualmente para paralelizar o código.

Em [Coons et al. 2008], os autores utilizam Aprendizagem por Reforço para alocar instruções e dados dentro de vários processadores. O trabalho apresenta uma utilização de RL dentro do contexto de otimizações de compiladores, porém o que os autores reduzem é o tempo gasto em comunicação.

No trabalho do DeepMind [Mnih et al. 2013], os autores usam RL de uma maneira mais tradicional. Eles visam criar um agente inteligente capaz de jogar diversos jogos de Atari. Apesar de utilizar Aprendizagem por Reforço, o trabalho foca em um campo distante da paralelização automática de código.

No artigo [Marinkovic et al. 2018], os autores propõem um algoritmo de paralelização automática de código em nível de instrução. O trabalho foca na paralelização automática de código, mas em nível de código de máquina binário.

Este trabalho se difere dos anteriores, pois utiliza Aprendizagem por Reforço como mecanismo para obter a paralelização de um determinado código, utilizando as diretivas OpenMP. Já os trabalhos anteriores utilizam técnicas de Aprendizagem de Má- 
quina para auxiliar o compilador no seu processo de tomada de decisão.

\section{Análise Estática e Paralelização de Código}

A Análise Estática de Código consiste como em conjunto de algoritmos que tem como principal objetivo extrair informações de um programa sem executá-lo [Cousot and Cousot 1977]. Este tipo de análise permite ao compilador fazer alterações e otimizações no programa sendo compilado a fim de aprimorá-lo. Um destes aprimoramentos é a paralelização do código sendo otimizado [Moreira et al. 2016]. Os três principais tipos de análises estáticas são: Dataflow Analysis, Constraint-Based Analysis e Type Analysis.

O Dataflow Analysis permite programar a informação através dos elementos de um programa baseando na suas dependências, que são definidas pela sintaxe da linguagem [Kam and Ullman 1977]. Estas análises são utilizadas para extrair informações dos elementos de um programa, por exemplo, identificar quando um valor atribuído a uma variável não seja mais necessário. Algumas análises que utilizam o fluxo de informação do programa são: Liveness, Available Expressions, Very Busy Expressions e Reaching Definitions.

O Constraint-Based Analysis se baseia na definição de restrições lógicas para um programa, ou seja, estas restrições não são definidas pela sintaxe do programa. Este tipo de análises é essencial para realizar o Control Flow Analysis [Allen 1970] que permitem a criação de um Control Flow Graph que envolve vários procedimentos em linguagens com dinamic dispatching. Essa estrutura de dados especifica o fluxo de execução de um programa e é a utilizada por várias outras análises. Este tipo de análise é o mais utilizado quando se realiza a paralelização automática [Moreira et al. 2016].

Na Type Analysis propaga-se informação usando os tipos anotados em um programa, esses tipos permitem provar propriedades para um programa [Jensen et al. 2009]. Type Analysis dependem de um sistema de tipos bem definido e da inferências destes tipos. Um exemplo deste tipo de análise são as garantias de segurança de memória da linguagem Rust [Klabnik and Nichols 2019].

\section{Aprendizagem por Reforço}

A Aprendizagem por reforço (RL) é um campo de Aprendizagem de Máquina inspirado na psicologia comportamental. Este campo estuda a criação de agentes inteligentes que tomam decisões em um ambiente com o objetivo de maximizar o acúmulo de recompensas recebidas a longo prazo [Mnih et al. 2013].

A maneira mais comum de modelar um problema de RL é utilizando um Processo de decisão de Markov (MDP) [Davis 2018], um framework matemático utilizado para modelar situações e processos de decisões nos quais as ações são parte estocásticos e parte determinísticos. A Figura 1 mostra uma representação gráfica de um MDP. Ele é utilizado na modelagem de problemas de otimização que podem ser resolvidos utilizando RL ou programação dinâmica [Sarkale et al. 2018]. Um MDP pode ser definido matematicamente como uma quíntupla $(S, A, P .(.,),. R .(.,),. \gamma))$ :

- $S$ : Conjunto de estados, pode ser finito ou infinito.

- A: Conjunto de ações que podem ser tomados pelo agente. 


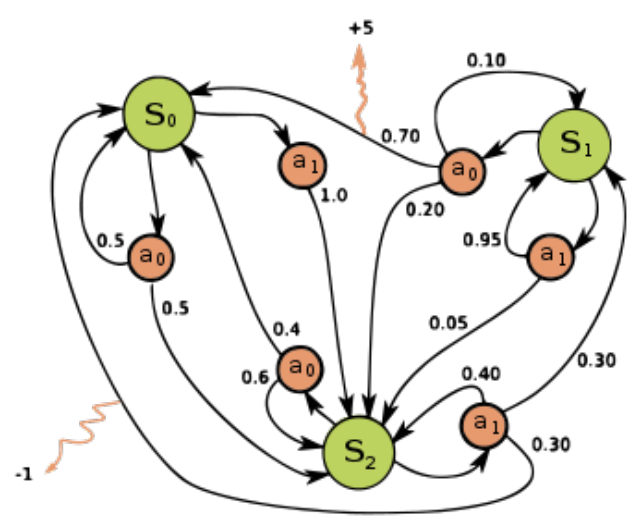

Figura 1. Exemplo de MDP.

- $P_{a}\left(s, s^{\prime}\right)=\operatorname{Pr}\left(s_{t+1}=s^{\prime} \mid s_{t}=s, a_{t}=a\right)$ : Função que determina a probabilidade de em um determinado momento no tempo $t$ estando no estado $s$ e tomando a ação $a$ o agente transitar para o estado $s^{\prime}$.

- $R_{a}\left(s, s^{\prime}\right)$ : Função que determina a recompensa imediata recebida pelo agente por ter realizado a ação $a$ em um estado $s$ e ter transitado para o estado $s^{\prime}$.

- $\gamma \in[0,1]$ : constante conhecida como Fator de Desconto. Determina a diferença de importância entre recompensas recentes e futuras.

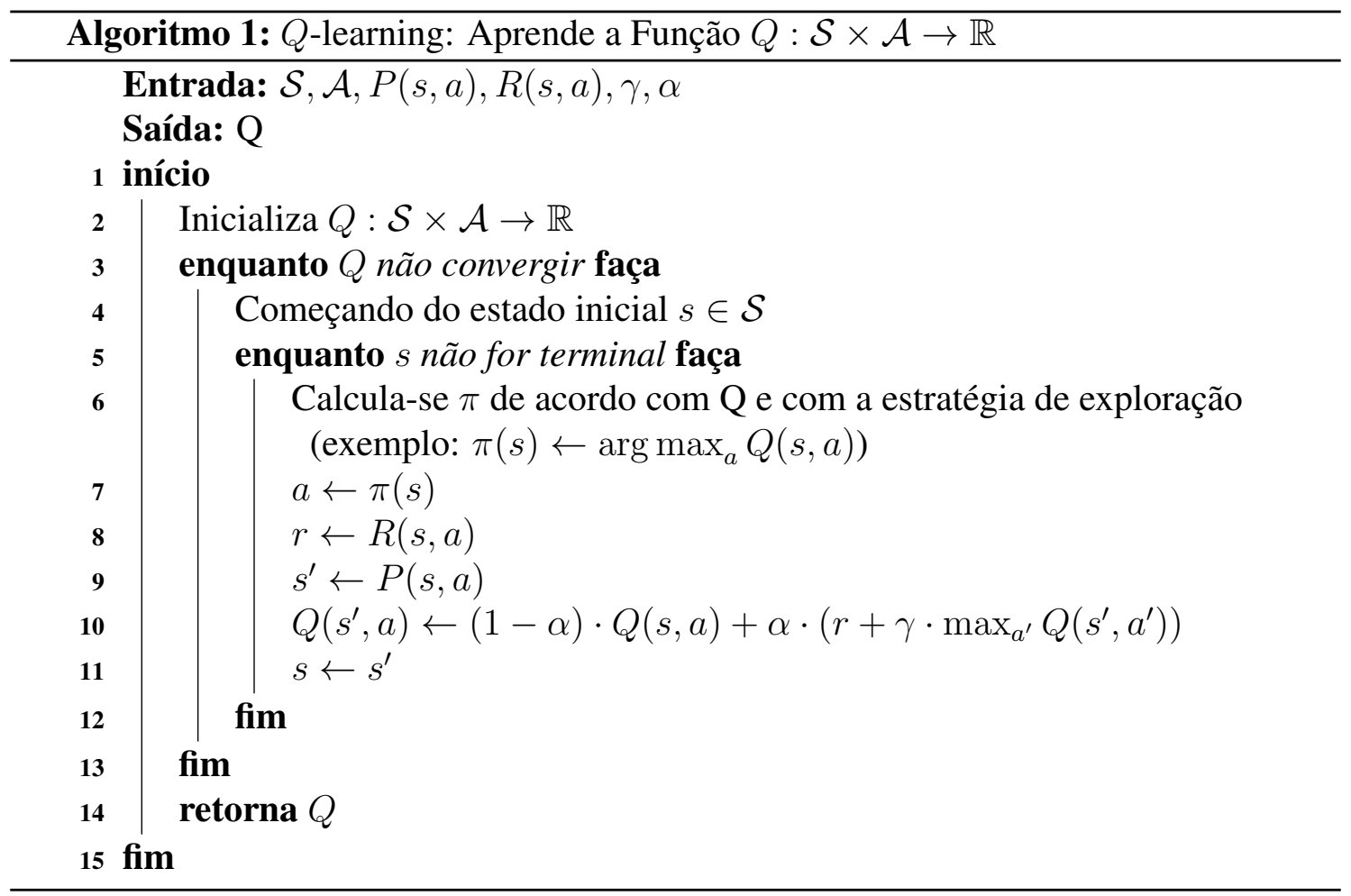

Uma vez definido o MDP, é necessário escolher um algoritmo capaz de determinar uma política, isto é, um mapeamento de qual ação tomar em cada estado para maximar o total de recompensas recebidas. Um algoritmo utilizado para isso é o QLearning [Mnih et al. 2013]. Este algoritmo aprende uma distribuição de probabilidade para 
cada estado do MDP, chamada de função $Q$, esta distribuição indica quais ações são mais prováveis de retornar a maior recompensa, este processo pode ser visto no Algoritmo 1.

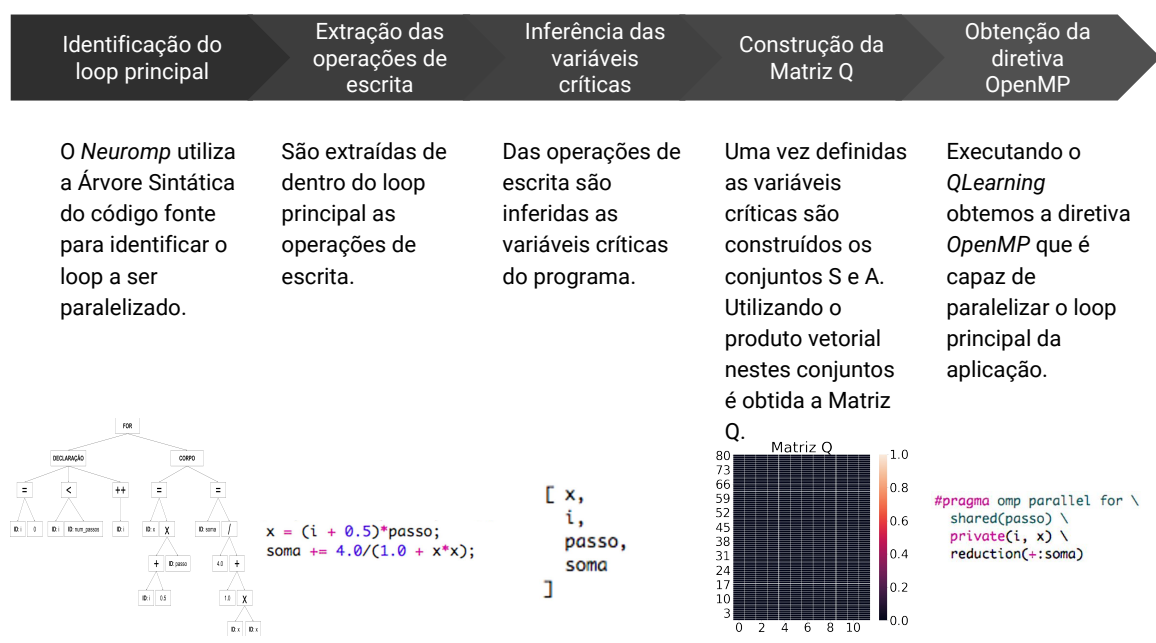

Figura 2. Funcionamento do NeurOMP.

\section{NeurOMP}

Nesta seção, é discutido o NeurOMP, uma otimização, aplicável em instruções do tipo for loop, que utiliza o algoritmo QLearning [Mnih et al. 2013] para definir a diretiva OpenMP capaz de paralelizar um algoritmo escrito na Liguagem C. Vale ressaltar que o NeurOMP realiza a paralelização em nível de dados e não trata condições que necessitam de exclusão mútua. O funcionamento do NeurOMP está exemplificado na Figura 2 .

O primeiro passo para se utilizar um algoritmo de aprendizagem por reforço é definir um MDP. Este arcabouço matemático nos permite definir os comportamentos tanto do mundo quanto do agente, desta forma cria-se um ambiente onde o agente pode aprender a maximizar o recebimento de recompensas a longo prazo. Para o NeurOMP, o MDP está definido da seguinte forma:

- $S$ : Conjunto das diretivas OpenMP válidas para um determinado loop.

- $A$ : Todas as transformações de variáveis permitidas pelo OpenMP.

- $P_{a}\left(s, s^{\prime}\right)=\operatorname{Pr}\left(s_{t+1}=s^{\prime} \mid s_{t}=s, a_{t}=a\right)$ : Essa função é constante, sendo a probabilidade igual a 1, uma vez que esse ambiente é totalmente determinista.

- $R_{a}\left(s, s^{\prime}\right)$ : Esta função está definida na Figura 4. Para o cálculo da recompensa são definidas 4 variáveis derivadas de um estado: $T_{s} s$ tempo de execução do código sequencial, $T_{s} p$ tempo de execução do código paralelo gerado em um estado $s$, $R_{s} s$ resultado da execução do código sequencial e $R_{s} p$ resultado da execução do código paralelo gerado em um estado $s$.

- $\gamma \in[0,1]$ : Este valor foi definido de maneira empírica em 0.95 .

O próximo passo para a execução do NeurOMP é a definição da matriz Q, utilizada pelo QLearning. Essa matriz é definida por $Q:(S \times A)$. Para obtermos $S$ e $A$, é necessário percorrer a Árvore Sintática e identificar a operação de loop principal da aplicação. Esta é indicada pelo programador usando uma diretiva similar do OpenMP. 


$$
R_{a}\left(T_{s} s, T_{s} p, R_{s} s, R_{s} p\right)= \begin{cases}-1, & T_{s} p>T_{s} s \\ -1, & R_{s} p \neq R_{s} s \\ \operatorname{speedup}\left(T_{s} s, T_{s} p\right), & \left(T_{s} s, T_{s} p, R_{s} s, R_{s} p\right) \in \mathbb{R}\end{cases}
$$

Figura 3. Equação que define a recompensa recebida em um determinado estado após tomar uma ação.

Uma vez determinado o loop, o NeurOMP identifica as variáveis críticas, variáveis que podem estar envolvidas em alguma condição de corrida. Isto é feito percorrendo mais uma vez a AST, dentro do loop, encontrando as variáveis envolvidas em operações de escrita. Com elas é possível definir os conjuntos $S$ e $A$ necessários na matriz Q.

Uma vez construída a matriz, executa-se o QLearning. O algoritmo utiliza as informações contidas no MDP para encontrar qual a diretiva OpenMP capaz de tornar o código paralelo. Porém, este método pode ser demorado para o processo de compilação. Por isso, é utilizado no NeurOMP, uma heurística para parar a execução caso o resultado já encontrado seja satisfatório. O programador pode definir um limiar para o speedup, caso o NeurOMP encontre uma paralelização que tenha um ganho de desempenho maior que o limiar, para-se a execução do QLearning.

Após a finalização da execução do NeurOMP, tem-se a função Q definida. Desta maneira, partindo do estado inicial da matriz Q e executando as melhores ações para cada estado, obtém-se a diretiva que gerou maior ganho de desempenho, do QLearning.

\section{Resultados Experimentais}

Para avaliar a eficiência do NeurOMP, foram realizados testes utilizando o CAP Bench [Souza et al. 2017]. Uma vez que este benchmark já é paralelizado com OpenMP, é comparado o desempenho da otimização com o especialista. Outra comparação de desempenho realizada é com as técnicas já existentes no compilador para paralelização automática de código. Para isso foram utilizados 2 compiladores, o ICC e o GCC, ambos utilizam análise estática para tentar realizar a paralelização. Em especial, o ICC utiliza diretivas de compilação para identificar quais loops paralelizar, estratégia similar ao NeurOMP.

Os testes foram realizados em uma arquitetura contendo dois processadores Intel Core i5, 8GB de memória principal, rodando o sistema operacional Linux Ubuntu 16.04. Para cada aplicação presente no CAP Bench, foram geradas 3 variações do código: uma versão sequencial, a versão paralelizada pelo humano e a versão paralelizada pelo NeurOMP. Foram realizados 2 testes, um para cada compilador. Os testes consistem em: compilar cada variação, paralelizar o código pelo compilador e por fim calcular o speedup em relação ao código sequencial para as paralelizações do compilador, do humano e do NeurOMP. Cada experimento foi executado por um total de 1000 interações, cada experimento levou em torno de 30 segundos para convergir. Cada aplicação foi executada 10 vezes em cada variação, a média aritmética e o desvio padrão foram calculados, garantindo-se um grau de confiança de $95 \%$.

Como pode ser visto na Figura 5, o NeurOMP foi capaz de paralelizar todas as aplicações do CAP Bench de maneira similar ao especialista humano. Apesar de alguns casos os compiladores conseguirem um desempenho superior, por eles paralelizarem to- 


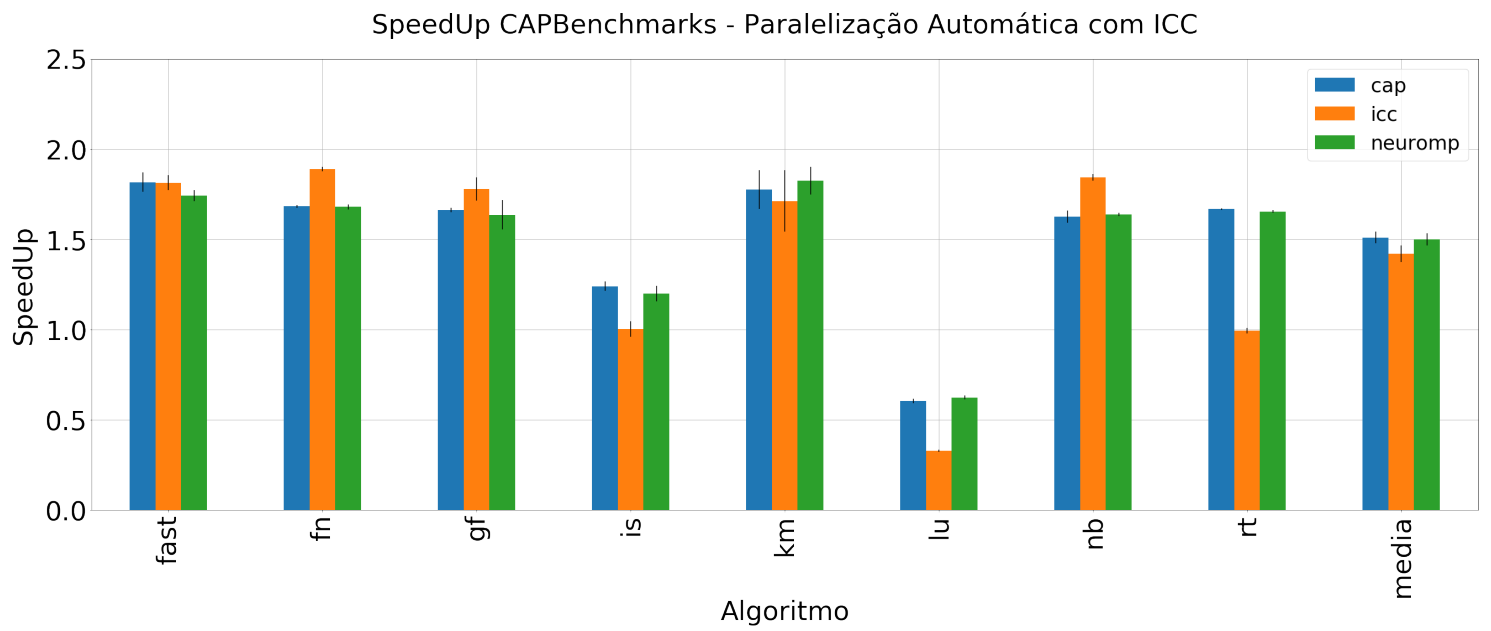

(a) Resultados com o ICC.

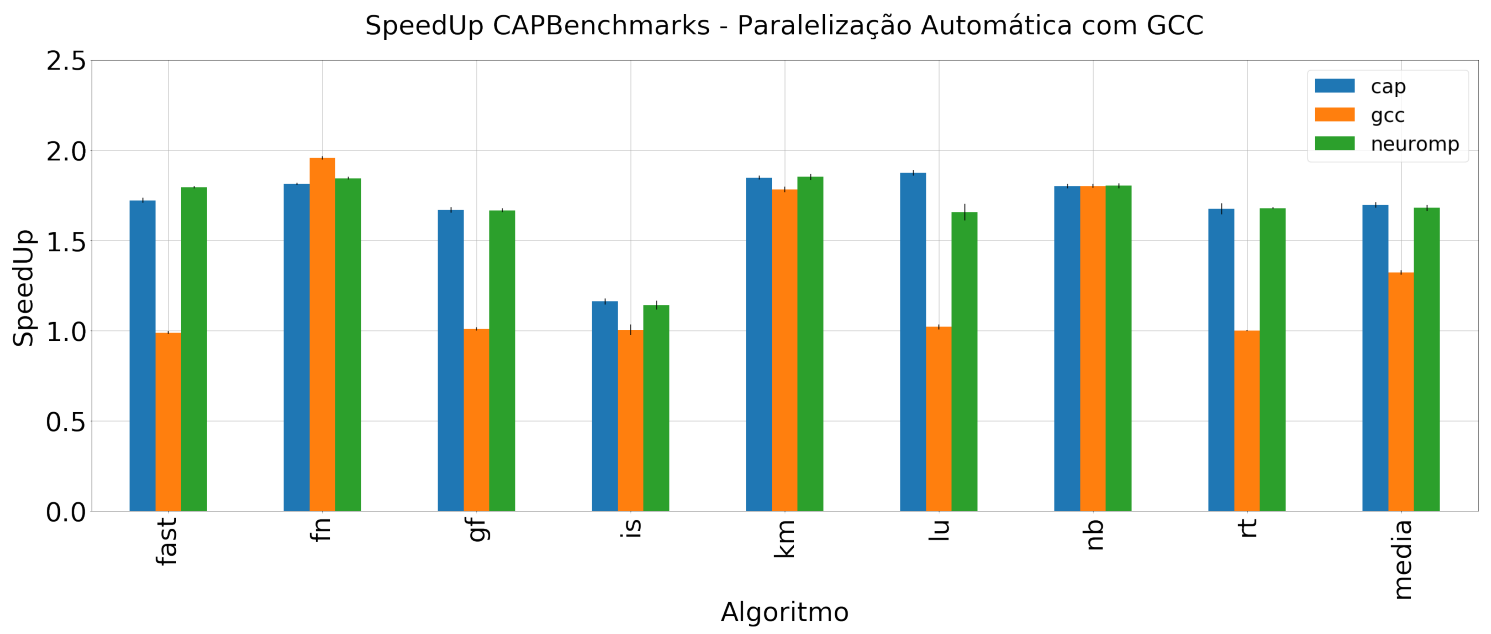

(b) Resultados com o GCC.

Figura 4. Resultados com os compiladores.

dos os loops enquanto o humano e o NeurOMP agem somente naqueles computacionalmente mais custosos. Nota-se na Figura 5, que para a paralelização da aplicação $L U$ no ICC, obteve-se um speedup inferior a 1, isso se deve ao fato que o compilador utilizou alguma outra otimização além do paralelismo que tornou o código sequencial significativamente mais rápido, tornando o paralelismo ineficiente. Como o ICC não possui código aberto, não é possível afirmar com certeza qual é esta otimização.

O NeurOMP obteve um ganho médio superior a ambos os compiladores, como poder ser visto na Figura 5. O speedup médio da otimização foi superior no GCC, em comparação com o ICC. Isto se deve ao fato que o ICC realiza mais otimizações do que o GCC, o que torna o código mais eficiente. Esta diferença do código gerado pode ser observada pela diferença de speedup nos dois compiladores. Pelos gráficos, o NeurOMP obteve um ganho de desempenho $27.19 \%$ maior em comparação ao GCC e $5.57 \%$ maior que o ICC. 


\section{Conclusão}

Neste trabalho, foi apresentado o NeurOMP, uma otimização que utiliza o QLearning, um algoritmo de aprendizagem por reforço, para realizar a paralelização automática de for loops utilizando OpenMP. Os resultados experimentais demonstram que a otimização obtém um speedup médio no CAP Bench igual a 1.6, similar a um especialista humano. Para trabalhos futuros, pode-se aplicar a mesma técnica para paralelizar para outras arquiteturas, como GPU ou FPGAs ou aglomerados de computadores. Além disso, pode estender o NeurOMP para que ele possa ser capaz de identificar regiões críticas e realizar paralelismo de tarefas.

\section{Referências}

Ahmed, J., Siyal, M. Y., Najam, S., and Najam, Z. (2017). Challenges and issues in modern computer architectures. In Fuzzy Logic Based Power-Efficient Real-Time MultiCore System, pages 23-29. Springer.

Allen, F. E. (1970). Control flow analysis. ACM Sigplan Notices, 5(7):1-19.

Bessey, A., Block, K., Chelf, B., Chou, A., Fulton, B., Hallem, S., Henri-Gros, C., Kamsky, A., McPeak, S., and Engler, D. (2010). A few billion lines of code later: using static analysis to find bugs in the real world. Communications of the ACM, 53(2):66-75.

Chapman, B., Jost, G., and Van Der Pas, R. (2008). Using OpenMP: portable shared memory parallel programming, volume 10. MIT press.

Coelli, T. J. (1996). A guide to frontier version 4.1: a computer program for stochastic frontier production and cost function estimation. Technical report, CEPA Working papers.

Coons, K. E., Robatmili, B., Taylor, M. E., Maher, B. A., Burger, D., and McKinley, K. S. (2008). Feature selection and policy optimization for distributed instruction placement using reinforcement learning. In PACT, pages 32-42. ACM.

Cousot, P. and Cousot, R. (1977). Abstract interpretation: a unified lattice model for static analysis of programs by construction or approximation of fixpoints. In Proceedings of the 4th ACM SIGACT-SIGPLAN symposium on Principles of programming languages, pages 238-252. ACM.

Dagum, L. and Menon, R. (1998). Openmp: an industry standard api for shared-memory programming. IEEE CSE, 5(1):46-55.

Davis, M. H. (2018). Markov models \& optimization. Routledge.

Fursin, G., Miranda, C., Temam, O., Namolaru, M., Yom-Tov, E., Zaks, A., Mendelson, B., Bonilla, E., Thomson, J., Leather, H., et al. (2008). Milepost gcc: machine learning based research compiler. In GCC Summit.

Gubner, T. and Boncz, P. (2017). Exploring query execution strategies for jit, vectorization and simd. In Eighth International Workshop on Accelerating Analytics and Data Management Systems Using Modern Processor and Storage Architectures (ADMS).

Jensen, S. H., Møller, A., and Thiemann, P. (2009). Type analysis for javascript. In International Static Analysis Symposium, pages 238-255. Springer. 
Kam, J. B. and Ullman, J. D. (1977). Monotone data flow analysis frameworks. Acta informatica, 7(3):305-317.

Khatami, Z. (2017). Compiler and Runtime Optimization Techniques for Implementation Scalable Parallel Applications. PhD thesis, LSU.

Klabnik, S. and Nichols, C. (2019). The Rust Programming Language (Covers Rust 2018). No Starch Press.

Kulkarni, S. and Cavazos, J. (2012). Mitigating the compiler optimization phase-ordering problem using machine learning. ACM SIGPLAN Notices, 47(10):147-162.

Marinkovic, V., Popovic, M., and Djukic, M. (2018). An automatic instruction-level parallelization of machine code. Advances in Electrical and Computer Engineering, 18(1):27-36.

Mnih, V., Kavukcuoglu, K., Silver, D., Graves, A., Antonoglou, I., Wierstra, D., and Riedmiller, M. (2013). Playing atari with deep reinforcement learning. arXiv preprint arXiv:1312.5602.

Moreira, K. C. A., Mendonça, G. S. D., Guimarães, B., Alves, P., and Pereira, F. M. Q. (2016). Paralelização automática de código com diretivas openacc. SBLP. SBC.

Sarkale, Y., Nozhati, S., Chong, E. K., Ellingwood, B., and Mahmoud, H. (2018). Solving markov decision processes for network-level post-hazard recovery via simulation optimization and rollout. arXiv preprint arXiv:1803.04144.

Schardl, T. B., Moses, W. S., and Leiserson, C. E. (2017). Tapir: Embedding fork-join parallelism into llvm's intermediate representation. In Proceedings of the 22nd ACM PPoPP, pages 249-265. ACM.

Souza, M. A., Penna, P. H., Queiroz, M. M., Pereira, A. D., Góes, L. F. W., Freitas, H. C., Castro, M., Navaux, P. O., and Méhaut, J.-F. (2017). Cap bench: a benchmark suite for performance and energy evaluation of low-power many-core processors. CCPE, 29(4).

Stephenson, M., Amarasinghe, S., Martin, M., and O'Reilly, U.-M. (2003). Meta optimization: improving compiler heuristics with machine learning. In ACM SIGPLAN Notices, volume 38, pages 77-90. ACM.

Tong, Y., Zhang, W., Ma, Y.-C., Liu, Y., Liang, Y., Zhang, T., and Luo, H. (2017). Compiler-guided parallelism adaption based on application partition for power-gated ilp processor. VLSI, 25(4):1329-1341.

Triantafyllis, S., Vachharajani, M., Vachharajani, N., and August, D. I. (2003). Compiler optimization-space exploration. In CGO 2003., pages 204-215. IEEE. 